\title{
Ultrastructure of Mycobacterium marinum granuloma in striped bass Morone saxatilis
}

\author{
D. T. Gauthier ${ }^{1, *}$, W. K. Vogelbein ${ }^{1}$, C. A. Ottinger ${ }^{2}$ \\ ${ }^{1}$ Department of Environmental and Aquatic Animal Health, Virginia Institute of Marine Science, \\ College of William and Mary, Gloucester Point, Virginia 23062, USA \\ ${ }^{2}$ United States Geological Survey, Leetown Science Center, National Fish Health Research Laboratory, \\ Kearneysville, West Virginia 25430, USA
}

\begin{abstract}
An emerging epizootic of mycobacteriosis currently threatens striped bass Morone saxatilis populations in Chesapeake Bay, USA. Several species of mycobacteria, including Mycobacterium marinum, species resembling $M$. avium, $M$. gordonae, $M$. peregrinum, $M$. scrofulaceum and $M$. terrae, and the new species $M$. shottsii have been isolated from diseased and healthy bass. In this study, we describe the ultrastructure of developing $M$. marinum granulomas in experimentally infected bass over a period of $45 \mathrm{wk}$. The primary host response to injected mycobacteria was formation of large macrophage aggregations containing phagocytosed bacilli. M. marinum were always contained within phagosomes. Close association of lysosomes with mycobacterial phagosomes, as well as the presence of electron-opaque material within phagosomes, suggested phagolysosomal fusion. Development of granulomas involved epithelioid transformation of macrophages, followed by appearance of central necrosis. Desmosomes were present between mature epithelioid cells. The necrotic core region of $M$. marinum granulomas was separated from overlying epithelioid cells by several layers of flattened, electron-opaque spindle-shaped cells. These cells appeared to be formed by compression of epithelioid cells and, aside from a flattened nucleus, did not possess recognizable organelles. Following the development of well-defined, paucibacillary granulomas, secondary disease was observed. Recrudescence was marked by bacterial replication followed by disruption of granuloma architecture, including loss of epithelioid and spindle cell layers. In advanced recrudescent lesions, normal tissue was replaced by macrophages, fibroblasts, and other inflammatory leukocytes. Large numbers of mycobacteria were observed, both intracellular and suspended in cellular debris.
\end{abstract}

KEY WORDS: Mycobacteriosis · Striped bass · Morone saxatilis · Mycobacterium marinum • Granuloma · Ultrastructure

\section{INTRODUCTION}

Granulomas are chronic inflammatory lesions composed of various cell types, including macrophages, lymphocytes, fibroblasts and granulocytes. Granuloma morphology varies widely, depending on eliciting agent, host species and other factors, but lesions are typically organized in concentric cellular layers, with a central region of macrophages surrounded by a collar of various other inflammatory cells. Macrophages in the interior of the granuloma differentiate into characteristic forms known as epithelioid (epithelial-like) cells (Sutton \& Weiss 1966, Papadimitriou \& Spector
1971). Epithelioid cells appear by hematoxylin and eosin (HE) staining as polygonal cells with large, pale nuclei and eosinophilic, faintly granular cytoplasm. These cells are usually tightly apposed with indistinct cytoplasmic boundaries, forming a solid mass of tissue.

Granulomas are most frequently elicited by agents that are resistant to intracellular degradative processes (Stenger et al. 1967, Papadimitriou \& Spector 1972, Black \& Epstein 1974, Doenhoff 1997). There are 2 major types of granulomas, depending on the eliciting agent. Foreign-body granulomas are formed in response to inert particles, such as talc or material from surgical sutures (Cotran et al. 1999). Typically, foreign- 
body granulomas are composed of a solid mass of epithelioid cells and giant cells, with the eliciting agent often visible in the center. The other major granuloma type, the immune granuloma, is formed in response to insoluble particles that stimulate a cell-mediated immune response, such as mycobacteria. Cytokines produced by $\mathrm{T}$ cells, such as IL-12 and interferon- $\gamma$, play an important role in formation and maintenance of immune granulomas (Cooper et al. 1993, Ehlers et al. 2000). A prominent feature of immune granulomas produced by mycobacteria such as Mycobacterium tuberculosis is caseous necrosis in the lesion core, which produces a highly acidic, anoxic environment that may serve to degrade otherwise refractory bacilli.

The structure and development of mycobacterial granulomas in mammals, especially those produced in response to Mycobacterium tuberculosis and BCG (attenuated $M$. bovis), have been described (Papadimitriou \& Spector 1972, Adams 1976, Browett et al. 1979). Мycobacterial granulomas in poikilotherms are less well understood, as few controlled temporal morphological studies have been performed. In the light of recent interest in M. marinum infections of fishes as model systems for exploring mycobacterial pathogenesis (Talaat et al. 1998, 1999, Davis et al. 2002), detailed study of the structure and development of teleost granulomas is necessary.

In a previous study (Gauthier et al. 2003), we described the histologic progression of experimental mycobacterial disease in striped bass over a period of $45 \mathrm{wk}$. Lesions produced in response to injected Mycobacterium marinum displayed a consistent morphological progression from early inflammatory cell aggregations to well-developed epithelioid granulomas with necrotic cores. In addition, disintegration of granulomas and subsequent secondary disease was observed. In this study, we examined this morphological progression at the ultrastructural level.

\section{MATERIALS AND METHODS}

The present work used tissues collected concomitantly with histologic and bacteriologic samples in a previous laboratory exposure study. Detailed methods of fish maintenance, mycobacterial infection, and sampling interval are given in Gauthier et al. (2003). Briefly, striped bass Morone saxatilis ( $\mathrm{n}=130$, mean weight $\pm \mathrm{SE}=116.7 \pm 3.9 \mathrm{~g})$ were infected intraperitoneally with $2.6 \times 10^{5}$ (long-term study, 8 to $45 \mathrm{wk}$ ) or $1.1 \times 10^{6}$ (short-term study, 2 to $8 \mathrm{wk}$ ) Mycobacterium marinum. Fish were sacrificed at 2, 4, 6, 8, 17, 26, 36, and $45 \mathrm{wk}$ post-injection (p.i.) for histologic and bacteriologic sampling. Samples of spleen, mesentery and anterior kidney were taken for ultrastructural examination from 3 fish at each sampling point.
Early time-point samples. In a third study, fish were injected with $1.4 \times 10^{6}$ Mycobacterium marinum as described previously (Gauthier et al. 2003). At 24 and $72 \mathrm{~h}$ p.i., fish were lavaged for peritoneal cells. We injected $10 \mathrm{ml}$ ice-cold L-15 (Liebovitz) medium with $100 \mathrm{U} \mathrm{ml}^{-1}$ sodium heparin (Sigma) into the peritoneal cavity and withdrew it through a ventral incision $10 \mathrm{~min}$ later. Cells were centrifuged at $400 \times g$ for 10 min, washed once in L-15, and fixed as described below.

Processing for electron microscopy. Tissues from fish sampled between 2 and 45 wk p.i. were fixed for 4 to $6 \mathrm{~h}$ in ice-cold $2 \%$ glutaraldehyde $/ 2.5 \%$ paraformaldehyde/0.15 M sucrose/0.1 M sodium cacodylate buffer ( $\mathrm{pH} 7.2$ ), followed by $1 \mathrm{~h}$ postfixation in $1 \%$ $\mathrm{OsO}_{4} / 0.1 \mathrm{M}$ sodium cacodylate. Cells obtained by peritoneal lavage were fixed for $30 \mathrm{~min}$ in cold $1.5 \%$ glutaraldehyde/2 \% paraformaldehyde/0.15 M sucrose/0.1 M sodium cacodylate ( $\mathrm{pH} 7.2$ ), and postfixed as described for tissue samples. Dehydration of both tissues and peritoneal cells was accomplished by a graded ethanol series with $1 \mathrm{~h}$ en bloc staining with saturated uranyl acetate in $70 \%$ ethanol. Alcohol was replaced by several changes of propylene oxide, after which tissues were infiltrated and embedded in Spurr's resin (Electron Microscopy Sciences). Ultrathin (90 nm) sections were cut on a Reichert-Jung ultramicrotome, mounted on carbon-stabilized Formvar-coated copper 1-hole grids, stained with Reynold's lead citrate (Reynolds 1963) for $7 \mathrm{~min}$ and examined on a Zeiss CEM 902 transmission electron microscope operating at $80 \mathrm{kV}$.

\section{RESULTS}

Cell types, organization, and ultrastructural features were similar in granulomas from spleen, anterior kidney and mesenteries, and were consistent in all fish sampled. The descriptions given below apply to developing granulomas in all 3 tissues except where specifically noted. Throughout, the term 'macrophage aggregation' is used to describe the early inflammatory response to mycobacteria. This term should not be confused with 'macrophage aggregate,' which is a focal accumulation of pigment-bearing macrophages in the spleen, anterior kidney or liver of many teleost fishes (Wolke 1992).

\section{Early macrophage aggregation (24 to $72 \mathrm{~h}$, lavage material)}

Large, roughly spherical aggregations of macrophages were observed in lavaged peritoneal cell preparations 24 and $72 \mathrm{~h}$ after injection with mycobacteria (Fig. 1a). 


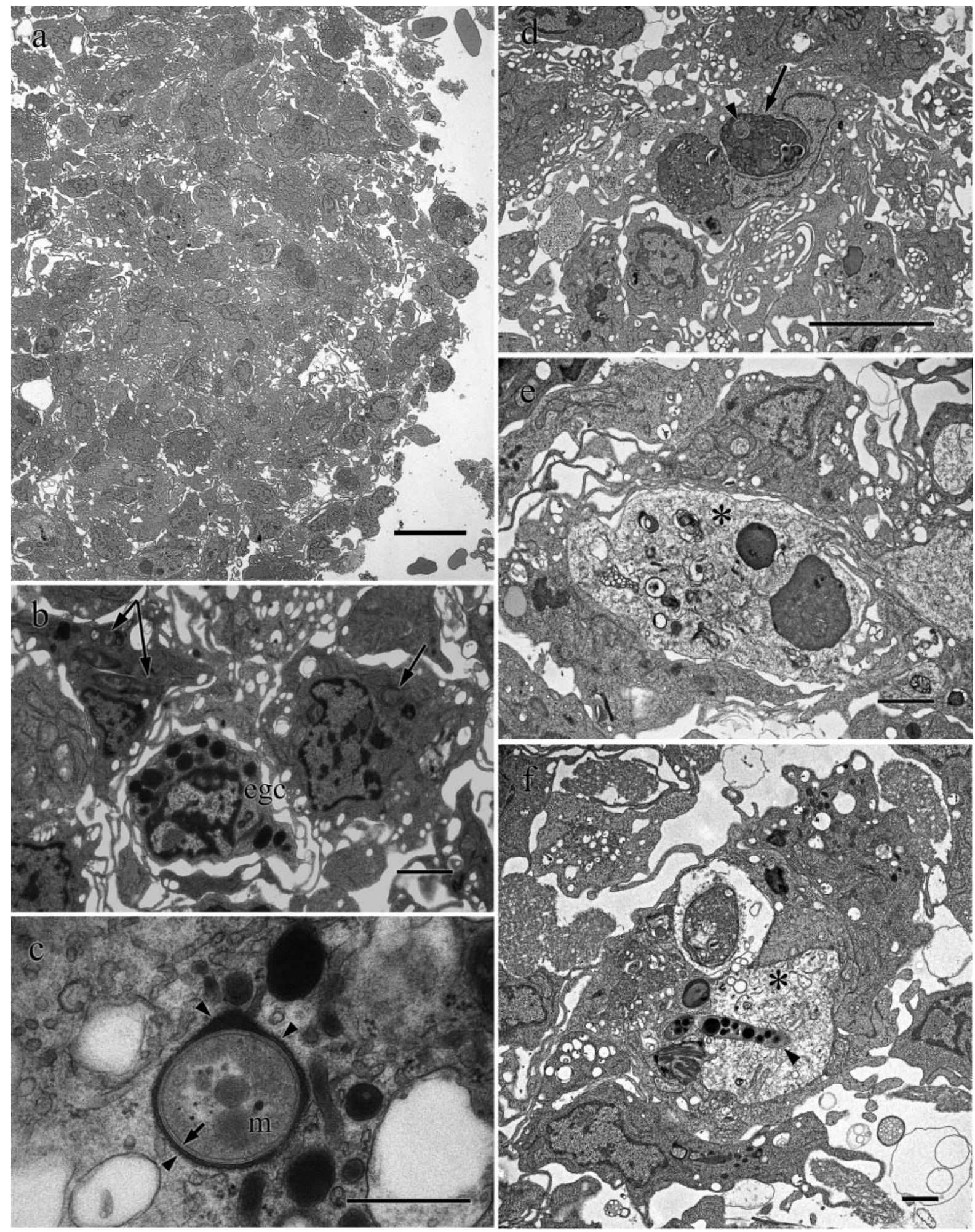

Fig. 1. Morone saxatilis injected with Mycobacterium marinum. (a) Aggregated macrophages. Scale bar = $10 \mu \mathrm{m}$. (b) Aggregated macrophages with phagocytosed mycobacteria (arrows) and eosinophilic granule cell (egc). Scale bar $=1 \mu \mathrm{m}$. (c) Transverse section of mycobacterium (m) within phagolysosome. Space between phagolysosomal membrane (arrowheads) and bacterial cell wall (arrow) is filled with electron-opaque material; phagolysosome is surrounded by electron-opaque lysosomes. Scale bar $=$ $0.5 \mu \mathrm{m}$. (d) Macrophage with electron-opaque phagosome (arrow) containing debris and a bacillus (arrowhead); note extensive filopodial interdigitations between adjacent cells. Scale bar $=5 \mu \mathrm{m}$. (e) Dead cell (*) being phagocytosed by adjacent macrophage(s). Scale bar $=1 \mu \mathrm{m}$. (f) Electron-lucent phagosome (*) containing debris and mycobacterium (arrowhead). Scale bar $=1 \mu \mathrm{m}$. 
The peripheral cytoplasm of aggregated cells formed long, slender filopodia that interdigitated with adjacent cells, while non-aggregated macrophages typically had shorter and broader pseudopodia. Organelle morphology of cells within aggregations and nonaggregated cells was similar at both times. Aggregated macrophages were polymorphic, ranging from 5 to $7 \mu \mathrm{m}$ in greatest dimension, and had ovoid to lobular nuclei with eccentric nucleoli (Fig. 1b). Cytoplasm was finely granular. Organelles included ovoid mitochondria, perinuclear Golgi apparatus, rough endoplasmic reticulum (rER), and spherical to ovoid membrane-limited endosomes of varying electron opacity. Endosomes were observed to fuse with phagosomes containing ingested material, and are thus collectively referred to as lysosomes, although their morphological variation suggests a degree of functional and/or developmental heterogeneity. Phagosomes contained cellular debris, myelin figures and/or mycobacteria.

All intracellular mycobacteria were contained within a limiting phagosomal membrane. Typically, phagosomes were tightly conformant to bacilli (Fig. 1b,c), but expanded phagosomes containing debris and mycobacteria were also observed (Fig. 1d,f). Electronopaque lysosomes were frequently observed in close proximity to mycobacterial phagosomes, and electronopaque material was often present in the space between the mycobacterial cell wall and phagosomal membrane (Fig. 1c). Cell death was observed frequently within macrophage aggregations, and dead cells were phagocytosed by other macrophages (Fig. 1e). When dead cells containing 1 or more bacilli were phagocytosed, expanded, electron-lucent phagosomes containing mycobacteria and debris resulted (Fig. 1f).

Non-aggregated peritoneal exudate cells included macrophages, eosinophilic granule cells (EGCs), neutrophilic granule cells (NGCs) and lymphocytes. EGCs were readily identifiable by large, homogeneous electron-opaque granules, and neutrophils were differentiated from morphologically similar macrophages by the presence of characteristic striated granules. All cell types were morphologically consistent with previous reports (Bodammer 1986, Bodammer \& Robohm 1996). Mycobacteria were rarely observed within nonaggregated macrophages. Occasional granulocytes were observed within macrophage aggregations (Fig. 1b).

\section{Inflammatory focus/epithelioid granuloma ( 2 to $8 \mathrm{wk}$ )}

The macrophage component of early mesenteric inflammatory foci was morphologically similar to macrophage aggregations from lavage samples. In addition to these cells, granulocytes and lymphocytes were present within and surrounding inflammatory foci. Large quantities of extracellular collagen microfibrils were observed within and surrounding large mesenteric inflammatory foci at 2 wk post-infection. Collagen was always accompanied by cells with dense cytoplasm, lobular to crenulate nuclei, extensive swollen rER, and few mitochondria. These cells were putatively identified as fibroblasts based on their consistent association with extracellular collagen and highly synthetic appearance. Fibroblast cell shape was variable, with both rounded and highly elongate forms observed.

Early macrophage aggregations rapidly developed into organized, stratified epithelioid granulomas. Stratified granulomas were present in the mesenteries as early as $2 \mathrm{wk}$, and in all 3 sampled tissues at $6 \mathrm{wk}$. There were 3 morphologically distinct cellular layers visible in organized lesions: (1) epithelioid cell layer, (2) spindle cell layer, and (3) necrotic core (Fig. 2a). A transitional layer of compressed epithelioid cells between Layers 1 and 2 was occasionally present.

Epithelioid cells first appeared at the center of inflammatory foci, and constituted the majority of cells in the interior of developing granulomas. morphological forms intermediate between macrophages and epithelioid cells were commonly observed in early lesions, with cells becoming increasingly epithelioid in appearance toward the lesion center. Epithelioid cells formed the outer layers of granulomas with core necrosis. Ultrastructurally, epithelioid cells resembled macrophages, with increased cytoplasmic and nuclear volume and increased numbers of mitochondria and rER profiles. These cells were highly compressed, and cytoplasmic boundaries were indistinct (Fig. 2b). Bundles of intermediate filaments could often be observed in the cytoplasm of epithelioid cells. Unlike macrophages of earlier aggregations, epithelioid cells rarely contained phagosomes or mycobacteria. In addition to cytoplasmic interdigitations, numerous desmosomes (macula adherens) were formed between adjacent epithelioid cells (Fig. 2b,c). Desmosomes varied in length, typically ranging between 100 and $400 \mathrm{~nm}$, but single desmosomes of up to $1.2 \mu \mathrm{m}$ were observed. Typical desmosomal components of attachment plaques and tonofilaments were present; however, the structures were somewhat unusual in that cytoplasmic intermediate filaments generally ran parallel to desmosomes and were linked to attachment plaques by short perpendicular filaments. In addition, a dense intermediate line was not observed, whereas it is typically present in mammalian desmosomes (Ghadially 1988).

Spindle-cell layers appeared concomitantly with core necrosis. Histologically these cells were highly com- 

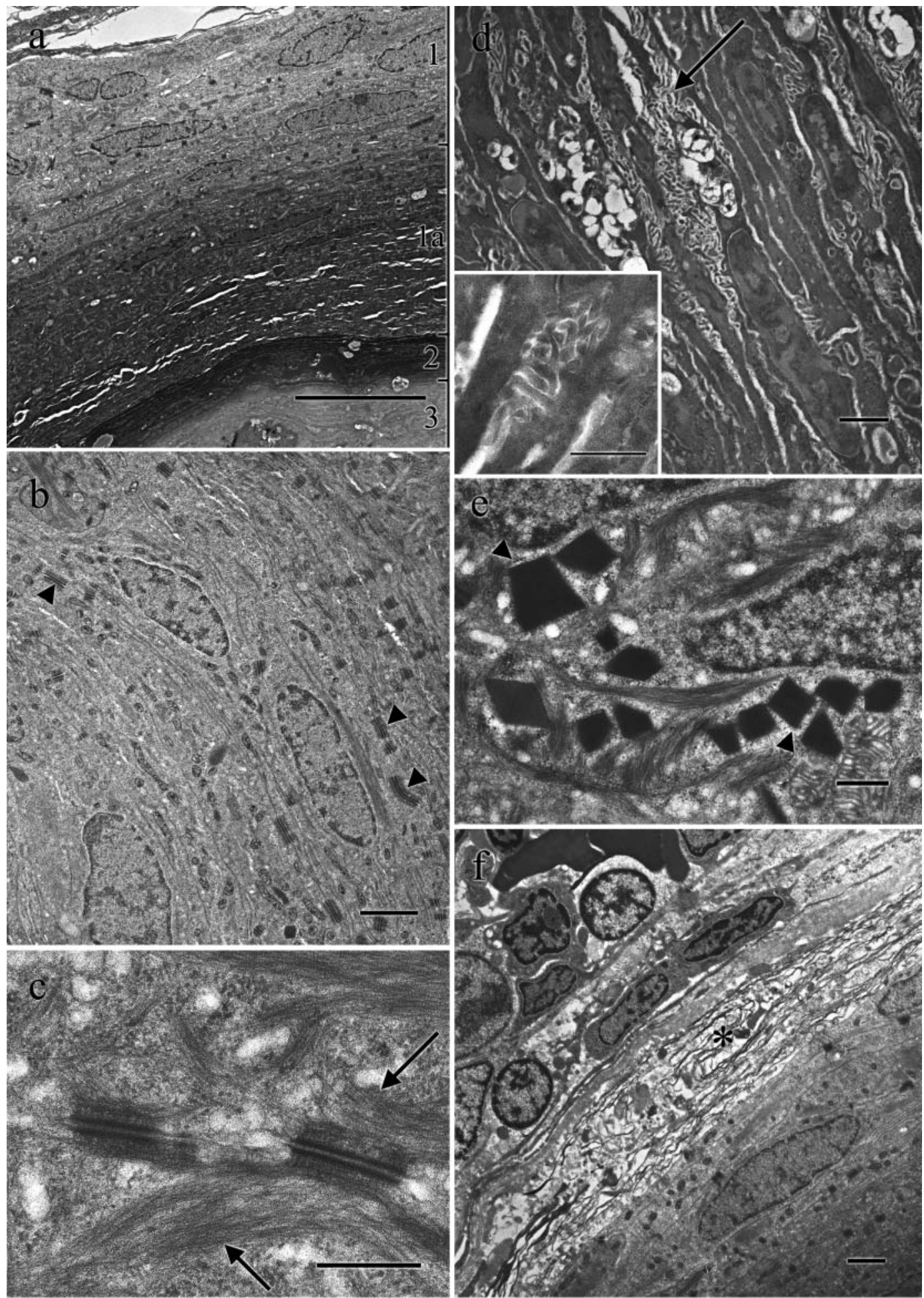

Fig. 2. Morone saxatilis injected with Mycobacterium marinum. (a) Granuloma stratification. Epithelioid (1), compressed epithelioid (1a), spindle-cell (2), and core layers (3) are visible (spleen, $8 \mathrm{wk}$ p.i.). Scale bar $=5 \mu \mathrm{m}$. (b) Epithelioid macrophages; multiple desmosomes (arrowheads) join adjacent cells (anterior kidney, $17 \mathrm{wk}$ p.i.). Scale bar $=1 \mu \mathrm{m}$. (c) Desmosomes between epithelioid cells; tonofilaments (arrows) are joined to electron-opaque attachment plaques via short perpendicular filaments (spleen, 8 wk p.i.). Scale bar $=$ $0.5 \mu \mathrm{m}$. (d) Spindle cells; note short fimbrial interdigitations (arrow, inset) (mesentery, $8 \mathrm{wk}$ p.i.). Scale bars $=1$ and $0.5 \mu \mathrm{m}$ (inset). (e) Crystalloid inclusions (arrowheads) within epithelioid cell (spleen, 8 p.i.). Scale bar $=0.5 \mu \mathrm{m}$. (f) Granuloma boundary; epithelioid cells (lower right) are separated from splenic parenchyma (upper left) by fibrous tissue (*) (spleen, 8 wk p.i.). Scale bar = $1 \mu \mathrm{m}$ 
pressed and intensely eosinophilic (Gauthier et al. 2003). Ultrastructurally, spindle cells were highly compressed, with electron-opaque cytoplasm and no recognizable organelles aside from flattened, heterochromatic nuclei (Fig. 2d). Cellular boundaries were indistinct, and adjacent cells elaborated extensive arrays of short interlocking fimbrial cytoplasmic projections (Fig. 2d and inset). In some lesions the boundary between epithelioid cells and spindle cells was sharply defined, whereas in others a gradual morphological progression to the spindle-cell state was observed.

Necrotic core regions were composed largely of amorphous, relatively electron-lucent debris with occasional identifiable cell fragments. Ultrastructural observation of mycobacteria corresponded with histologic findings (Gauthier et al. 2003). Bacilli were very rare in core material of granulomas from fish that tested negative for acid-fast bacilli by the Ziehl-Neelsen technique.

Crystalloid inclusions were frequently observed within all layers of granulomas, especially at later stages in development (Fig. 2e). Crystalloids were irregular, trigonal to hexagonal, measured 0.2 to $0.7 \mu \mathrm{m}$ between apices, and had no discernible internal periodicity. In macrophages and early epithelioid cells, crystalloids were generally contained within a phagosome or fine limiting membrane, whereas crystalloids in mature epithelioid cells, spindle cells and core material appeared to be free in the cytoplasm or extracellular. Similar crystalloid structures were observed within intact and degranulating EGCs surrounding granulomas. Crystalloids were highly eosinophilic by hematoxylin and eosin stain.

Granulomas were typically surrounded by a thick capsular matrix of collagen microfibrils. In early epi- thelioid granulomas, EGCs, lymphocytes and macrophages of varying epithelioid character were present throughout the deposited collagen. These cells were somewhat polymorphic and often conformed tightly to the surrounding matrix. The cellularity of the collagenous layer generally decreased with the development of core necrosis and spindle cells, so that in wellstratified granulomas the outer layers of epithelioid cells were separated from normal tissue parenchyma by an acellular fibrous matrix (Fig. 2f).

\section{Bacillary granuloma/recrudescence}

When present in large numbers within intact granulomas, bacteria were located within core and spindle cell layers. Core bacilli were suspended in necrotic material. Due to extreme compression and the lack of distinct limiting membranes, it could not be definitively determined if bacilli in the spindle cell layer were intra- or extra-cellular (Fig. 3a). Transverse division of individual bacilli was commonly observed, and large concentrations of bacteria were often seen at the interface between spindle cells and necrotic core material. Both intact mycobacteria and structures suggestive of degrading bacilli were observed in core and spindle cell layers (Fig. 3b).

Disruption of bacillary granulomas was marked by a loss of lesion stratification, disappearance of spindle cell layers, a ragged outer margin of epithelioid cells, and the reappearance of inflammatory cells on the lesion periphery (Fig. 4a). Rather than the compressed, tightly joined concentric layers observed in earlier granulomas, epithelioid cells were oriented in several
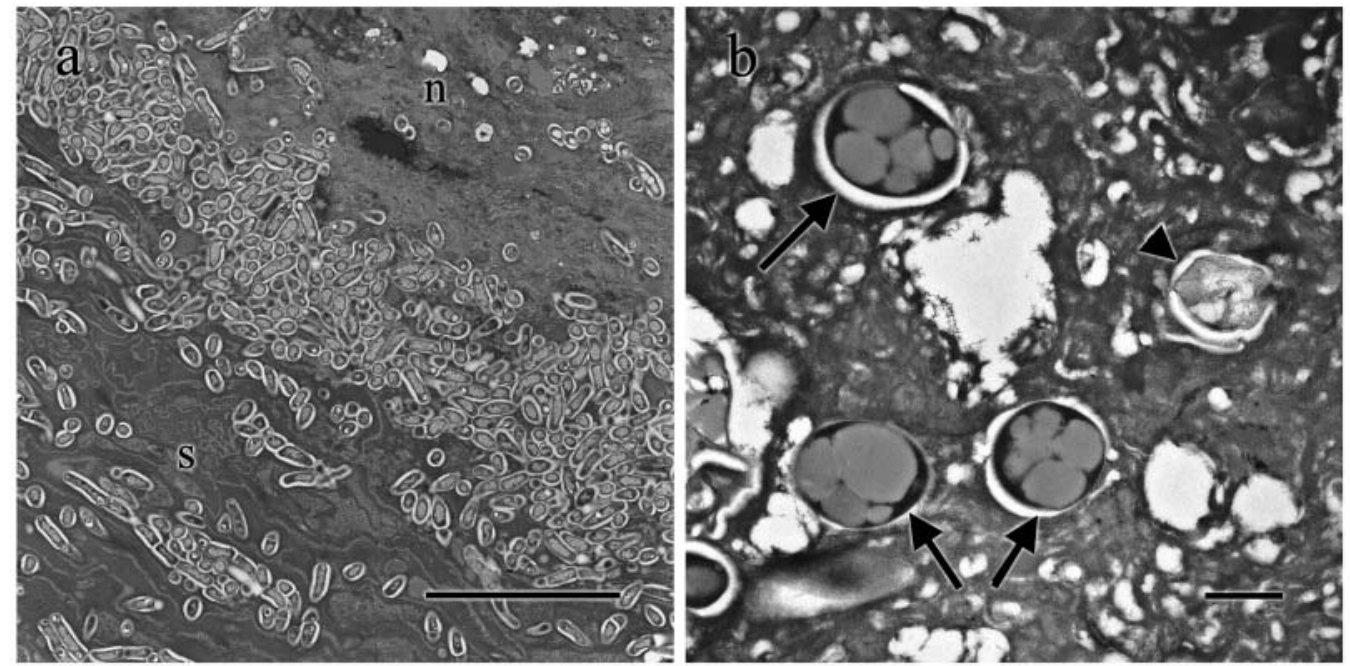

Fig. 3. Morone saxatilis injected with Mycobacterium marinum. (a) Mycobacteria within necrotic material (n) and spindle cell layer (s) of granuloma (mesentery, $36 \mathrm{wk}$ p.i.). Scale bar $=5 \mu \mathrm{m}$. (b) Intact (arrows) and possible degenerating (arrowhead) $M$. marinum in core material; electron transparent zone surrounding mycobacteria is probably artifactual (anterior kidney, $17 \mathrm{wk}$ p.i.). Scale bar $=0.5 \mu \mathrm{m}$ 

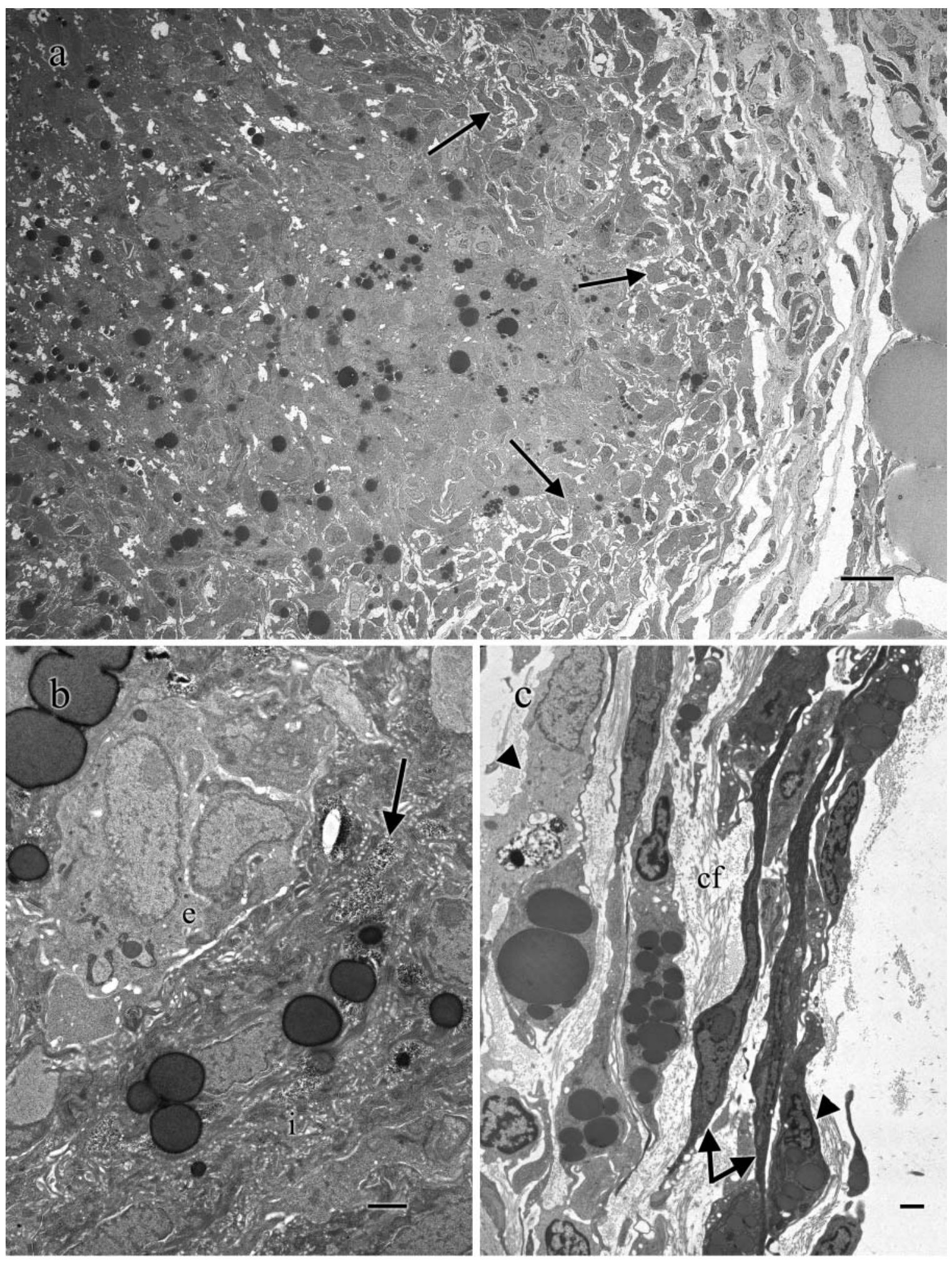

Fig. 4. Morone saxatilis injected with Mycobacterium marinum. (a) Disrupted granuloma; note ragged edge of epithelioid layer (arrows) and absence of lesion zonation. Scale bar $=10 \mu \mathrm{m}$. (b) Lesion interior; cells with epithelioid appearance (e) and cells with morphology intermediate between epithelioid and spindle cells (i) are present; electron-opaque granular deposits are present between cells (arrow). Scale bar $=1 \mu \mathrm{m}$. (c) Lesion margin; matrix of collagen microfibrils (cf) is populated with fibroblasts (arrows) and macrophages (arrowheads) of varying electron-opacity. Lipid droplets are common in macrophages. Scale bar $=1 \mu \mathrm{m}$. All mesentery, $32 \mathrm{wk}$ p.i. 

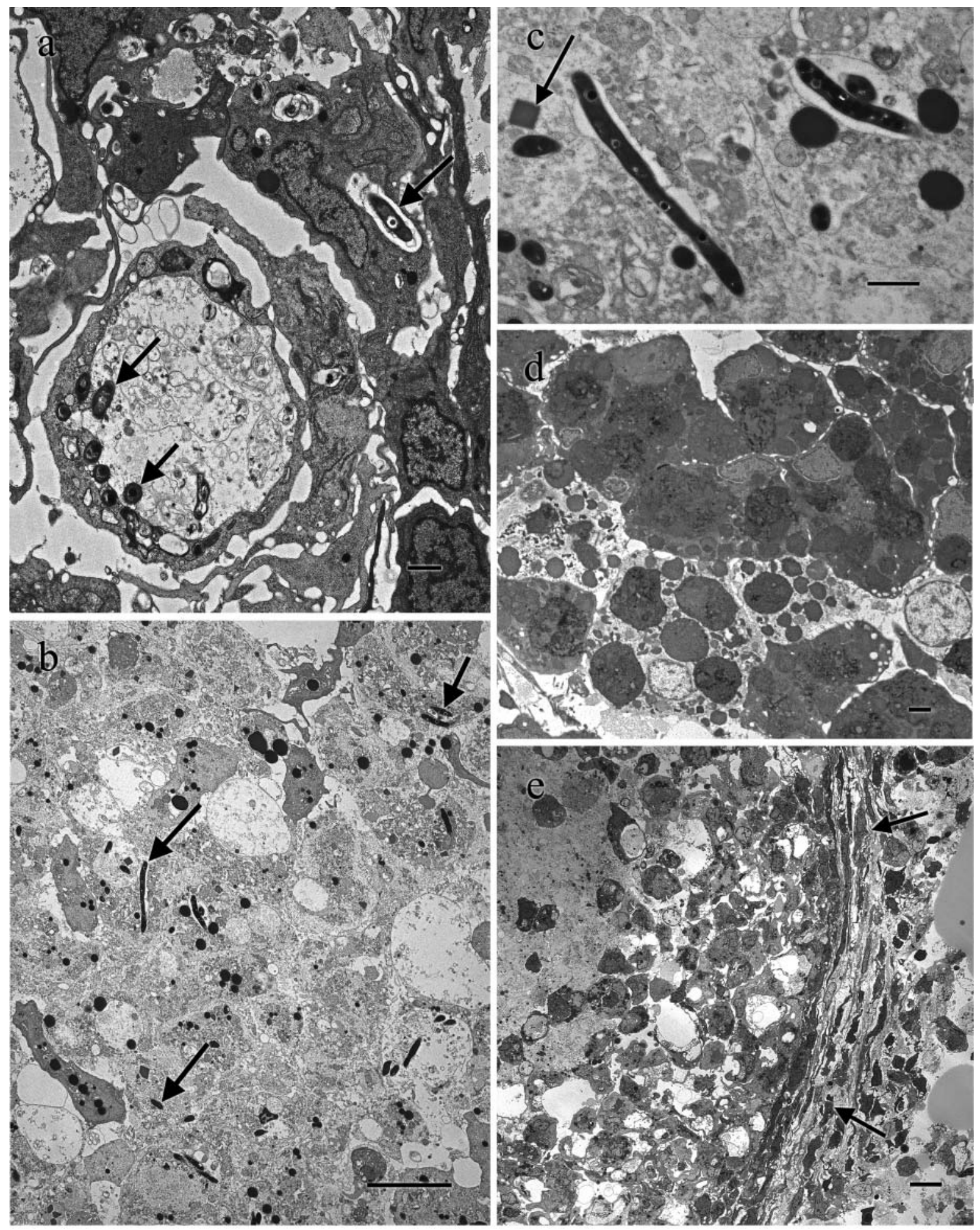

Fig. 5. Morone saxatilis injected with Mycobacterium marinum. (a) Advanced recrudescent lesion. Granuloma organization is lost and normal tissue is replaced with inflammatory leukocytes and/or dead cells. Mycobacteria (arrows) are present within both conformant and expanded, debris-containing phagosomes. Scale bar $=1 \mu \mathrm{m}$. (b) Intact mycobacteria (arrows) among degraded cellular remnants. Scale bar $=10 \mu \mathrm{m}$. (c) Detail of mycobacteria in cellular debris; note presence of crystalloids (arrow) and residual membrane. Scale bar $=1 \mu \mathrm{m}$. (d) Debris-replete and degrading macrophages. Scale bar $=1 \mu \mathrm{m}$. (e) Granuloma remnant; fibrous/cellular shell remains (arrows), but epithelioid cells of interior have been replaced with loose organization of inflammatory leukocytes and necrotic material. Scale bar $=10 \mu \mathrm{m}$. All anterior kidney, 45 wk p.i., except (e) which is spleen, 45 wk p.i. 
different directions and not as tightly apposed. Toward the center of lesions, cells had relatively electronopaque cytoplasm and few organelles. Intercellular pockets of granular, electron-opaque material were frequently observed (Fig. 4b). Necrotic material and numerous mycobacteria were observed in the core regions of disrupted lesions. The fibrous capsule of disrupted lesions was often highly cellular, with macrophages, granulocytes and fibroblasts (Fig. 4c).

Secondary disease was marked by replacement of granulomas with a poorly organized, loose reticular network of macrophages, lymphocytes, fibroblasts and granulocytes. Epithelioid cells were rare or absent from these lesions. Intracellular mycobacteria in intact macrophages were contained within both tightly conformant and expanded, debris-containing phagosomes (Fig. 5a). Transverse division of bacteria within phagosomes was commonly observed. Large areas of dead cells with bacilli were present in some lesions, as were concentrations of debris-swollen macrophages (Fig. 5b,c,d). A residual membrane was frequently observed around bacilli suspended in cellular debris (Fig. 5c). Granuloma remnants in varying degrees of disintegration were occasionally observed (Fig. 5e). The interior of these remnants was typically replaced by macrophages and other inflammatory leucocytes, as well as diffuse necrotic material.

\section{DISCUSSION}

Histologic development of granulomas in Mycobacterium marinum-infected striped bass has been described previously (Gauthier et al. 2003). This study expands those findings to the ultrastructural level and provides information on the very early ( 24 to $72 \mathrm{~h}$ ) cellular response to injected mycobacteria. Formation of the $M$. marinum granuloma in striped bass Morone saxatilis appeared to be effected primarily by macrophages, which were capable of considerable polymorphism in various lesion layers. Lymphocytes, fibroblasts and granulocytes were also present at all stages of granuloma development. Membrane contact between these cells and macrophages was common, and macrophages often contained products apparently derived from EGCs (i.e. crystalloids). These observations suggest a role for accessory cells in the formation and maintenance of granulomas in fishes.

Intracellular Mycobacterium marinum in macrophage aggregations and early epithelioid granulomas were always bounded by a limiting phagosomal membrane. In many cases, mycobacteria appeared to be contained within fused phagolysosomes, based on electron-opaque material within the phagosome and apparent fusion events with surrounding lysosomes
(Fig. 1c). This observation is surprising, in the light of the paradigm that mycobacteria, including $M$. marinum, survive intracellularly in vitro by inhibiting phagosome-lysosome fusion of host macrophages (Armstrong \& Hart 1971, Barker et al. 1997). Morphological evidence of in vivo phagolysosomal fusion has also been presented for long-term $M$. marinum granulomas in frogs, and it has been suggested that macrophage processing of mycobacteria may differ in vitro and in vivo (Bouley et al. 2001). Preliminary findings by our group, however, indicate that fusion of lysosomes with M. marinum phagosomes also occurs in vitro (data not shown). Additional experiments are currently underway to resolve this issue.

Epithelioid cells, so called because of their histologic resemblance to true epithelial cells, are derived in mammals from the monocyte/macrophage lineage. The transformation of mammalian monocytes into epithelioid cells is well documented in vivo (Papadimitriou \& Spector 1971, Adams 1974, Browett et al. 1979), and has been demonstrated in vitro for avian monocytes (Sutton \& Weiss 1966). Whether piscine epithelioid cells derive from the same lineage is less clear, especially considering their unusual capability to elaborate desmosomes. Desmosomes are generally considered to be characteristic of true epithelial or mesothelial cells (Ghadially 1988), although desmosome-like structures have been observed between histiocytes in certain human dermatopathies, as well as rat carageenan granuloma (Caputo \& Gianotti 1979, Monis \& Valentich 1986). Noga et al. (1989) also observed the presence of desmosomes in piscine granulomatous inflammation, and postulated that either (1) the capacity of macrophages to elaborate desmosomes is a primitive characteristic that has been lost in avian and mammalian orders, or (2) the cells involved in certain types of piscine inflammation derive from a nonmacrophage lineage (i.e. mesothelial cells). The findings of the present study indicate that the epithelioid cells of Mycobacterium marinum granulomas in striped bass are derived from macrophages. Macrophages in the majority of granulomas displayed a continuum of morphological features between ameboid phagocyte and static, mature epithelioid cell, typically with increasing epithelioid characteristics toward the lesion core. Mature epithelioid cells were tightly packed and strongly linked by interdigitations and desmosomes, and migration of dissimilar cell types through the epithelioid layers was not observed. This arrangement implies sequential development of epithelioid cells from free macrophages, as well as transformation of epithelioid cells into spindle cells. In addition, we have observed desmosome formation between adjacent adherent peritoneal macrophages in vitro (unpubl.). These results strongly suggest that myco- 
bacterial granulomas in fishes are, in fact, composed of differentiated macrophages, as in mammals, and that piscine macrophages are capable of desmosome formation. Thus, desmosome formation appears to be a primitive characteristic of macrophages. Whether the human macrophage and the functionally and morphologically analogous cell termed 'macrophage' in fishes arise from an homologous lineage, however, remains to be determined.

Mammalian epithelioid cells in BCG granulomas have reduced phagocytic capacity, but retain a pinocytic and excretory capability, the ability to synthesize DNA and RNA, and the ability to divide (Papadimitriou \& Spector 1971, 1972). The maintenance of endosomes, ER, Golgi apparatus and numerous mitochondria in the epithelioid cells of striped bass granulomas indicates that they may also play an active role in granuloma maintenance and function. Mitotic figures among epithelioid cells or granulomaassociated macrophages were rare histologically and were not observed ultrastructurally in the present study. Papadimitriou \& Spector (1971) have described epithelioid cell division as asymmetric cytokinesis resulting in an immature-appearing daughter macrophage. No immature-appearing cells were observed among the epithelioid cell layers in this study. These observations indicate that increases in granuloma cell number were due primarily to recruitment and differentiation of cells, rather than division of cells within the lesion. This is consistent with previous findings in rabbits and frogs (Ando \& Dannenberg 1972, Bouley et al. 2001). In epithelioid cells near the lesion core, organelles became less numerous and cytoskeletal elements more prominent, suggesting a role transition from metabolically active cell to structural sequestering element. Reduced metabolic activity in these cells would not be unexpected, considering that granulomas have no internal vascular elements, thus making cells in the lesion interior dependent on diffusion of oxygen through overlying cellular layers. Spindle cells bordering the lesion core had no recognizable organelles save a highly condensed nucleus, and therefore appeared metabolically inactive. The thickened, fimbrial structures observed between spindle cells differed markedly from the cellular interdigitations in overlying layers, however, suggesting that the transformation from mature epithelioid cells to spindle cells was not simply one of passive compression, but instead involved active formation or rearrangement of cellular elements.

Mycobacterium marinum granulomas were paucibacillary by both histologic and ultrastructural observation from the early epithelioid through wellorganized spindle cell stages. Whether the nidus of intra-granuloma bacterial replication lay within the core material or spindle cells was not determined by this study, although the distribution of bacteria in some lesions suggests rapid replication at the spindle cell-core interface and subsequent spread throughout both regions (Fig. 3a). Structures suggestive of degrading mycobacteria were also frequently observed within these regions (Fig. 3b). Death and degradation of mycobacteria in the lesion core would not be surprising, considering that the granuloma interior is generally considered to be hostile to bacterial life due to acidity, hypoxia, and the presence of hydrolytic enzymes. The presence of morphologically intact bacilli and the observed rapid outgrowth of bacteria in bacillary granulomas, however, indicated that a subpopulation of mycobacteria was capable of withstanding the environment of the inner granuloma. The mechanisms by which these few mycobacteria were able to survive, as well as the cues that led to the observed rapid bacterial outgrowth remain unknown at this time. Further study of $M$. marinum infection in striped bass may provide insights into mechanisms of latency and secondary disease for other pathogenic mycobacteria.

Latency of mycobacteria in both endothermic and poikilothermic animals remains poorly understood (Parrish et al. 1998, Flynn \& Chan 2001). It is known that pathogenic mycobacteria (e.g. Mycobacterium tuberculosis) may remain viable and infectious in asymptomatic individuals after primary infection (Opie \& Aronson 1927, Feldman \& Baggenstoss 1939). Acidfast staining frequently fails to reveal mycobacteria in healed primary infection foci, however, leading some to postulate the existence of chromophobic forms of mycobacteria via either alteration or loss of the cell wall (Harada 1977, Khomenko 1987). Indeed, varying degrees of acid-fast chromophobicity have been demonstrated for mycobacteria in situ and in vitro (Nyka \& O'Neill 1970, Dhople 1985), and we have found evidence for chromophobic mycobacteria within paucibacillary striped bass granulomas (Gauthier et al. 2003). No obvious differences in fine structure were observed for the rare mycobacteria within such lesions, however, and no alternate bacillary forms could be discerned. Therefore, this study did not find evidence for the existence of differing forms of mycobacteria within striped bass granulomas. Rather, it appeared that the large numbers of mycobacteria in bacillary granulomas arose from a small number of bacteria that survived within the central region of the lesions. No widely accepted ultrastructural description of altered or cell wall-deficient mycobacterial forms in situ exists, however, so hypotheses on their existence cannot be rejected on the basis of this study. Immunolocalization or in situ hybridization studies will be necessary to more clearly demonstrate the quantity, form and location of viable bacteria within striped bass granulomas. 
Numerous crystalloid eosinophilic inclusions were an unusual feature of the striped bass granuloma. Several types of spontaneously forming eosinophilic crystals have been described in the mammalian literature. Charcot-Leyden crystals (CLCs) are commonly described in human conditions of eosinophilia, such as allergy, parasitic infection, eosinophilic granuloma and various neoplasms (Charcot \& Robin 1853, Dvorak et al. 1990, Carson et al. 1992). CLCs are composed of the enzyme lysophospholipase (lysolecithin acylhydrolase, EC 3.1.1.5) (Weller et al. 1982), which spontaneously forms regular geometric structures, usually hexagonal bipyramids, when released from damaged or degranulating eosinophils (El-Hashimi 1971). Crystalline inclusions are also found in the core of most intact human eosinophil granules (Miller et al. 1966), although lysophospholipase has been ultrastructurally localized to a subpopulation of granules without crystalline cores (Dvorak et al. 1988). We have not observed crystalline cores within striped bass EGCs. Eosinophilic crystals of irregular shape isolated from deletional mutant and transgenic mice have been identified as Ym1, a protein of unknown function with in vitro chitinase activity (Guo et al. 2000). Crystals of this protein have been postulated to play a role in cell pathology during murine Cryptococcus neoformans infections (Feldmesser et al. 2001). Although the crystals associated with striped bass granulomas bear similarities in morphology and origin with the mammalian crystals mentioned, biochemical or immunological characterization will be necessary for definitive identification.

Acknowledgements. The authors thank P. Mason for technical assistance, and Drs. V. Blazer, C. Densmore, S. Kaattari, and F. Panek for critical review. This work was supported by US Geological Survey Cooperative Research Agreement \#01ERAG0015.

\section{LITERATURE CITED}

Adams DO (1974) The structure of mononuclear phagocytes differentiating in vivo. I. Sequential fine and histologic studies of the effect of Bacillus Calmette-Guerin (BCG). Am J Pathol 76:17-48

Adams DO (1976) The granulomatous inflammatory response. Am J Pathol 84:164-191

Ando M, Dannenberg AM (1972) Macrophage accumulation, division, maturation, and digestive and microbicidal capacities in tuberculous lesions. IV. Macrophage turnover, lysosomal enzymes, and division in healing lesions. Lab Investig 27:466-472

Armstrong JA, Hart PD (1971) Response of cultured macrophages to Mycobacterium tuberculosis, with observations on fusion of lysosomes with phagosomes. J Exp Med 134: 713-740

Barker L, George K, Falkow S, Small P (1997) Differential trafficking of live and dead Mycobacterium marinum organisms in macrophages. Infect Immun 65:1497-1504
Black MM, Epstein WL (1974) Formation of multinucleate giant cells in organized epithelioid cell granulomas. Am J Pathol 74:263-270

Bodammer JE (1986) Ultrastructural observations on peritoneal exudate cells from the striped bass. Vet Immunol Immunopathol 12:127-140

Bodammer JE, Robohm RA (1996) Ultrastructural observations on the phagocytic behavior of winter flounder Pleuronectes americanus peritoneal neutrophils and macrophages in vivo. Dis Aquat Org 25:197-208

Bouley DM, Ghori N, Mercer KL, Falkow S, Ramakrishnan L (2001) Dynamic nature of host-pathogen interactions in Mycobacterium marinum granulomas. Infect Immun 69: 7820-7831

Browett PJ, Simpson LO, Blennerhassett JB (1979) Experimental granulomatous inflammation: The ultrastructure of the granuloma induced by injection of the tubercle bacilli into Freund adjuvant-sensitised guinea-pigs. J Pathol 129: 191-200

Caputo R, Gianotti F (1979) Junctions between histiocytes: Role of coated vesicles. J Ultrastruct Res 68:256-264

Carson HJ, Buschmann RJ, Weisz-Carrington P, Choi YS (1992) Identification of Charcot-Leyden crystals by electron microscopy. Ultrastruct Pathol 16:403-411

Charcot JM, Robin C (1853) Observations de leucocythemie [Observations of leukocytosis]. C R Séances Soc Biol 5: $44-50$

Cooper A, Dalton D, Stewart T, Griffin J, Russell D, Orme I (1993) Disseminated tuberculosis in interferon gamma gene-disrupted mice. J Exp Med 178:2243-2247

Cotran RS, Kumar V, Collins T (1999) Pathologic basis of disease. WB Saunders, Philadelphia, PA

Davis JM, Clay H, Lewis JL, Ghori N, Herbomel P, Ramakrishnan L (2002) Real-time visualization of Mycobacteriummacrophage interactions leading to initiation of granuloma formation in zebrafish embryos. Immunity 17:693-702

Dhople AM (1985) Influence of prior periodic acid oxidation on the acid-fastness of Mycobacterium leprae. IRCS (Int Res Commun Syst) Med Sci 13:1259-1260

Doenhoff MJ (1997) A role for granulomatous inflammation in the transmission of infectious disease: schistosomiasis and tuberculosis. Parasitology 115:113-125

Dvorak AM, Letourneau L, Login GR, Weller PF, Ackerman SJ (1988) Ultrastructural localization of the Charcot-Leyden crystal protein (lysophospholipase) to a distinct crystalloid-free granule population in mature human eosinophils. Blood 72:150-158

Dvorak AM, Letourneau L, Weller PF, Ackerman SJ (1990) Ultrastructural localization of Charcot-Leyden crystal protein (lysophospholipase) to intracytoplasmic crystals in tumor cells of primary solid and papillary epithelial neoplasm of the pancreas. Lab Invest 62:608-615

Ehlers S, Kutsch S, Ehlers EM, Benini J, Pfeffer K (2000) Lethal granuloma disintegration in Mycobacteria-infected TNFRp $55^{-/}$mice is dependent on $\mathrm{T}$ cells and IL-12. J Immunol 165:483-492

El-Hashimi W (1971) Charcot-Leyden crystals. Am J Pathol 65:311-324

Feldman WH, Baggenstoss AH (1939) The occurrence of virulent tubercle bacilli in presumably non-tuberculous lung tissue. Am J Pathol 15:501-515

Feldmesser M, Kress Y, Casadevall A (2001) Intracellular crystal formation as a mechanism of cytotoxicity in murine pulmonary Cryptococcus neoformans infection. Infect Immun 69:2723-2727

Flynn JL, Chan J (2001) Tuberculosis: Latency and reactivation. Infect Immun 69:4195-4201 
Gauthier DT, Rhodes MW, Vogelbein WK, Kator H, Ottinger CA (2003) Experimental mycobacteriosis in striped bass (Morone saxatilis). Dis Aquat Org 54:105-117

Ghadially FN (1988) Ultrastructural pathology of the cell and matrix. Butterworths, London

Guo L, Johnson RS, Schuh JCL (2000) Biochemical characterization of endogenously formed eosinophilic crystals in the lungs of mice. J Biol Chem 275:8032-8037

Harada K (1977) Staining mycobacteria with periodic acidcarbol-pararosanilin: Principle and practice of the method. Microsc Acta 79:224-236

Khomenko AG (1987) The variability of Mycobacterium tuberculosis in patients with cavitary pulmonary tuberculosis in the course of chemotherapy. Tubercle 68:243-253

Miller F, de Harven E, Palade GE (1966) The structure of eosinophil leukocyte granules in rodents and in man. J Cell Biol 31:349-362

Monis B, Valentich MA (1986) Ultrastructure of macrophages of carrageenan granuloma in the rat with data on gamma-glutamyltranspeptidase activity. J Leukocyte Biol 39:133-140

Noga EJ, Dykstra MJ, Wright JF (1989) Chronic inflammatory cells with epithelial cell characteristics in teleost fishes. Vet Pathol 26:429-437

Nyka W, O'Neill EF (1970) A new approach to the study of non-acid-fast mycobacteria. Ann NY Acad Sci 174: 862-871

Opie EL, Aronson JD (1927) Tubercle bacilli in latent tuberculous lesions and in lung tissue without tuberculous lesions. Arch Pathol Lab Med 4:1-21

Papadimitriou JM, Spector WG (1971) The origin, properties

Editorial responsibility: David Bruno,

Aberdeen, UK and fate of epithelioid cells. J Pathol 105:187-203

Papadimitriou JM, Spector WG (1972) The ultrastructure of high- and low-turnover inflammatory granulomata. J Pathol 106:37-43

Parrish NM, Dick JD, Bishai WR (1998) Mechanisms of latency in Mycobacterium tuberculosis. Trends Microbiol 6:107-112

Reynolds ES (1963) The use of lead citrate at high pH as an electron-opaque stain in electron microscopy. J Cell Biol $17: 208-212$

Stenger RJ, Warren KS, Johnson EA (1967) An ultrastructural study of hepatic granulomas and schistosome egg shells in murine hepatosplenic Schistosomiasis mansoni. Exp Molec Pathol 7:116-132

Sutton JS, Weiss L (1966) Transformation of monocytes in tissue culture into macrophages, epithelioid cells, and multinucleated giant cells. J Cell Biol 28:303-331

Talaat AM, Reimschuessel R, Wasserman SS, Trucksis M (1998) Goldfish, Carassius auratus, a novel animal model for the study of Mycobacterium marinum pathogenesis. Infect Immun 66:2938-2942

Talaat AM, Trucksis M, Kane AS, Reimschuessel R (1999) Pathogenicity of Mycobacterium fortuitum and Mycobacterium smegmatis to goldfish, Carassius auratus. Vet Microbiol 66:151-164

Weller PF, Bach D, Austen KF (1982) Human eosinophil lysophospholipase: The sole protein component of Charcot-Leyden crystals. J Immunol 128:1346-1349

Wolke RE (1992) Piscine macrophage aggregates: A review. Annu Rev Fish Dis 2:91-108

Submitted: December 30, 2003; Accepted: April 15, 2004

Proofs received from author(s): October 12, 2004 